\title{
STUDY OF ANTIBIOTIC RESISTANCE PATTERN IN UROPATHOGENS AT A TERTIARY CARE HOSPITAL
}

Dr. Prasanna Gupta ${ }^{1}$.

1. Assistant Professor, Department of Microbiology, Konaseema Institute of Medical Sciences, Amalapuram, Andhra Pradesh, India.

\section{CORRESPONDING AUTHOR}

Dr. Prasanna Gupta ${ }^{1}$, Dept. of Microbiology,

Konaseema Institute of Medical Sciences,

Amalapuram- 533201, Andhra Pradesh, India,

E-mail: pramin1414@yahoo.co.in,

Ph: 09630238572.

ABSTRACT: BACKGROUND: Background and objective: Empiric treatment of urinary tract infections (UTI) is determined by the antibiotic sensitivity patterns of uropathogens in a population. There is increased resistance to the first line empirical drugs used in the treatment of urinary tract infection. This study was conducted to determine patterns of resistance amongst uropathogens in Amalapuram (India), to help establish local guidelines on treatment Of UTI. METHODOLOGY: This is a retrospective study on 323 urine cultures from July 2011 to June 2012. Antibiotic susceptibility testing was done by Kirby-Bauer disc diffusion method and compared. Analysis was done using simple percentage method. RESULTS: Out of the 323 samples subjected for culture, 152(47.06\%) were positive for growth. Out of the 152 culture isolates, E. coli was the most common (46.7\%) followed by Klebsiella spp (18.4\%), Candida spp (8.5\%), Staphylococcus aureus (7.8\%), Pseudomonas spp (6.5\%), Citrobacter $\operatorname{spp}(6.5 \%)$, Proteus spp (2.6\%), and Acinetobacter spp( 2.6\%). All bacterial isolates were $100 \%$ sensitive to Imipenem except Pseudomonas and Klebsiella which were $90 \%$ and $92.8 \%$ sensitive respectively. All the isolates were $100 \%$ resistant to Ampicillin except Staphylococci which was $16.6 \%$ sensitive. E. coli and Klebsiella were sensitive in range of $21.1 \%$ to $50 \%$ to Ciprofloxacin, Ceftriaxone, and Cefotaxime. Staphylococci were $100 \%$ sensitive to Vancomycin followed by 91.6\% sensitive to Linezolid, Cefotaxime and Amikacin. Acinetobacter and Proteus were having 100\% sensitivity to Amikacin and Ciprofloxacin. CONCLUSION: The alarming rate of resistance to Ciprofloxacin, Cefotaxime, Ceftriaxone, SXT and Ampicillin for major urinary isolate E. coli and Klebsiella, precludes the use of these commonly used antibiotics for empiric treatment of UTI in India. . Urine culture for screening and diagnosis is recommended.

KEY WORDS: Urinary tract infection, antibiotic susceptibility, India

INTRODUCTION: Urinary tract infection (UTI) is one of the most frequent conditions encountered by general practitioners .[1-3]An acute uncomplicated urinary tract infection (UTI) is one of the most common bacterial infections in women.[1-3] It is estimated that as many as $60 \%$ of all women report having had a UTI atleast once in their lifetime.[4,5] Worldwide, about 150 million people [6] are diagnosed with UTI each year, costing in excess of 6 billion dollars [7]. Among both outpatients and inpatients, Escherichia coli is the most common isolate, accounting for $75 \%$ to $90 \%$ of uncomplicated UTI isolates [8,9]. Staphylococcus saprophyticus, Klebsiella spp., Proteus spp., Enterococcus spp., and Enterobacter spp. are organisms less commonly isolated from outpatients. In the majority of cases, antibiotics are given empirically before the final bacteriology results are available. Therefore, area-specific 
monitoring studies to document the microorganisms causing UTI and their antimicrobial susceptibility is mandatory for helping the selection of an effective empirical treatment.[10]UTIs are often treated with different broad-spectrum antibiotics when one with a narrow spectrum of activity maybe appropriate because of concerns about infection with resistant organisms. Fluoroquinolones are preferred as initial agents for empiric therapy of UTI in area where resistance is likely to be of concern $[11,12]$. This is because they have high bacteriological and clinical cure rates, as well as low rates of resistance, among most common uropathogens[13-15]. The resistance pattern of community acquired UTI pathogens has not been studied extensively. [13]The extensive uses of antimicrobial agents have invariably resulted in the development of antibiotic resistance, which, in recent years, has become a major problem worldwide [16]. The etiology of UTI and the antibiotic resistance of uropathogens have been changing over the past years, both in community and nosocomial infection $[17,18]$. However, there is no much information on etiology and resistance pattern of community acquired UTIs in India. This retrospective study was done to compare the frequency and drug resistance pattern in uropathogens isolated from patients with UTIs in Amalapuram, India .

MATERIALS \& METHODS: This study was designated as a retrospective survey of 323 urine culture specimens from July 2011 to june 2012. As we had no control over collection of specimens, we excluded those culture isolates, which are likely to be contaminants, except those, which were isolated in 2 consecutive cultures. Cultures which yielded more than one isolate were excluded from the study group. All the culture isolates were identified in the department of Microbiology, KIMS, Amalapuram by standard laboratory techniques. Antimicrobial susceptibility testing was done by Kirby-Bauer disc diffusion method as per CLSI criteria. ATCC control strains(E.coli ATCC 25922, for Pseudomonas ATCC 27853 and for S.aureus ATCC 25923) were used as per CLSI guidelines. All the analysis was performed using simple percentage method.

RESULTS: In the present study total 323 samples were studied. Out of them 152(47.06\%) were culture positive. Out of the 152 culture isolates, E. coli was the major isolate, followed by Klebsiella spp (28), Candida spp(13), Staphylococcus aureus (12), Pseudomonas spp(10), Citrobacter spp(10), Proteus spp(4) and Acinetobacter spp(4). E. coli and Klebsiella spp were sensitive in range of $21.1 \%$ to $50 \%$ to Ciprofloxacin, Ceftriaxone, and Cefotaxime. All bacterial isolates were $100 \%$ sensitive to Imipenem except Pseudomonasspp and Klebsiella spp, which were $90 \%$ and $92.8 \%$ sensitive respectively. All the isolates were $100 \%$ resistant to Ampicillin except staphylococci which were $16.6 \%$ sensitive to Ampicillin. Staphylococci were $100 \%$ sensitive to Vancomycin followed by $91.6 \%$ sensitive to Linezolid and Cefotaxime and Amikacin .Acinetobacterspp and Proteus spp were having 100\% sensitivity to Amikacin and Ciprofloxacin. Pseudomonasspp, Staphylococcus aureus, Acinetobacter spp and Proteus spp were sensitive to Piperacillin- Tazobactum in a range of 75 to $83.3 \%$. Staphylococcus aureus, Pseudomonas spp and Citrobacter spp were also sensitive to ciprofloxacin in a range of 70 to $75 \%$. (Table - 2)

DISCUSSION: The most commonly isolated organism in our study was E. coli. The proportion of bacterial species isolated was similar to those described in several previous studies [19,20]. This study shows the distribution and antibiotic susceptibility pattern of microbial species isolated from patients with UTIs in KIMS, Amalapuram. These organisms cause a variety of infections including UTIs [21]. Most common age group affected in the present study was 21-30 
years $(31.57 \%)$ followed by $31-40$ years $(25.66 \%)$. Females $(76.97 \%)$ were more frequently affected than males(23.03\%).The age and sex distribution of the study group is shown in the table.3.

It has been extensively reported that adult women have a higher prevalence of UTI than men, principally due to anatomic and physical factors [22,23]. The UTIs are more frequent in women than in men, which corresponds to our findings because $76.97 \%$ of our patients were females. [24]

High resistance rates to the oral antibiotics in our study may be due to the uncontrolled consumption of these antibiotics in the community in the past decade in our region [25,26]. On the other hand, resistance to Amikacin, Piperacillin- Tazobactum and Meropenem are low, likely reflecting lower usage of these drugs. Our study demonstrates extremely low susceptibility to the first-line agents (Ampicillin, Ampicillin/ Sulbactum, Ciprofloxacin, Cotrimoxazole) in uropathogens in our population. However, recent studies have demonstrated therapeutic failure in more than $50 \%$ of patients infected with Cotrimoxazole resistant urinary pathogens $[27,28]$.

The worldwide trend of empirically treating UTI may not apply for specific geographical regions such as India, where decreased susceptibility rates are documented for common urinary pathogens. In the Indian setting, routine urine cultures may be necessary, since treatment failure with empirical therapy is likely to occur. International guidelines are no longer applicable for treating UTI in India, and development of specific guidelines based on local susceptibility patterns are necessary. Development of regional surveillance programs is necessary to provide information which would then enable the development of Indian UTI guidelines.

CONCLUSION: The alarming rate of resistance to Ciprofloxacin, Cefotaxime, Ceftriaxone, SXT and Ampicillin for major urinary isolates E. coli and Klebsiella spp, precludes the use of these commonly used antibiotics for empiric treatment of UTI in India. . Urine culture for screening and diagnosis purpose is recommended.

Table 1 Organisms isolated from UTI cases $(n=152)$

\begin{tabular}{|l|c|c|}
\hline \multicolumn{1}{|c|}{ Organisms } & No.of isolates & \% of isolation \\
\hline Escherichia Coli & 71 & 46.71 \\
\hline Klebsiella spp. & 28 & 18.42 \\
\hline Candida spp. & 13 & 8.55 \\
\hline $\begin{array}{l}\text { Staphylococcus } \\
\text { aureus }\end{array}$ & 12 & 7.89 \\
\hline $\begin{array}{l}\text { Pseudomonas } \\
\text { aeruginosa }\end{array}$ & 10 & 6.57 \\
\hline Citrobacter spp. & 10 & 6.57 \\
\hline Proteus spp. & 4 & 2.63 \\
\hline Acinetobacter spp. & 4 & 2.63 \\
\hline Total & 152 & 100 \\
\hline
\end{tabular}


Table2: ABST pattern of the isolates (in percentage)

\begin{tabular}{|c|c|c|c|c|c|c|c|c|}
\hline ANTIBIOTIC & $\begin{array}{l}\text { E. Coli } \\
(n=71 \\
)\end{array}$ & $\begin{array}{l}\text { Klebsi } \\
\text { ellasp } \\
\text { p. } \\
(n=28 \\
)\end{array}$ & $\begin{array}{l}\text { Staph.a } \\
\text { ureus } \\
(n=12)\end{array}$ & $\begin{array}{l}\text { Pseudom } \\
\text { onas spp. } \\
(n=10)\end{array}$ & $\begin{array}{l}\text { Citroba } \\
\text { cter } \\
\text { spp. } \\
(n=10)\end{array}$ & $\begin{array}{l}\text { Acinetob } \\
\text { acter } \\
\text { spp. } \\
(n=4)\end{array}$ & $\begin{array}{l}\text { Prot } \\
\text { eus } \\
\text { spp. } \\
(n=4 \\
\text { ) }\end{array}$ & $\begin{array}{l}\text { Candi } \\
\text { da } \\
\text { spp. } \\
(n=1 \\
3)\end{array}$ \\
\hline G & 30.9 & 42.8 & 83.3 & 30 & 40 & 50 & 60 & NT \\
\hline A & 0 & 0 & 16.6 & NT & 0 & 0 & 0 & NT \\
\hline AS & 40.8 & 25 & 25 & 20 & 30 & 25 & 50 & NT \\
\hline $\mathrm{AK}$ & 56.3 & 57.1 & 91.6 & 70 & 70 & 100 & 100 & NT \\
\hline $\mathrm{CA}$ & 28.1 & 46.4 & 58.3 & 40 & 50 & 75 & 50 & NT \\
\hline $\mathrm{CE}$ & 47.8 & 50 & 91.6 & 40 & 60 & 50 & 75 & NT \\
\hline $\mathrm{CI}$ & 38 & 53.5 & 83.3 & 30 & 60 & 50 & 75 & NT \\
\hline $\mathrm{CF}$ & 36.6 & 46.4 & 75 & 70 & 70 & 100 & 100 & NT \\
\hline SXT & 21.1 & 39.2 & 58.3 & 60 & 50 & 25 & 50 & NT \\
\hline I & 100 & 92.8 & 100 & 90 & 100 & 100 & 100 & NT \\
\hline $\mathrm{LZ}$ & NT & NT & 91.6 & NT & NT & NT & NT & NT \\
\hline NA & 49.2 & 28.5 & 0 & 0 & 40 & 50 & 50 & NT \\
\hline $\mathrm{NF}$ & 50.8 & 25 & 75 & NT & 50 & NT & NT & NT \\
\hline NX & 23.9 & 53.5 & 58.3 & 60 & 60 & 75 & 75 & NT \\
\hline $\mathrm{P}$ & NT & NT & 0 & NT & NT & NT & NT & NT \\
\hline VA & NT & NT & 100 & NT & NT & NT & NT & NT \\
\hline PT & 46.4 & 57.1 & 83.3 & 80 & 80 & 75 & 75 & NT \\
\hline
\end{tabular}


Table 3.shows age and sex distribution of the study group

\begin{tabular}{|c|c|c|c|}
\hline Age in years & MALES (\%) & FEMALES (\%) & Total (\%) \\
\hline 1 1-10 years & 2 & 3 & $5(3.29 \%)$ \\
\hline $11-20$ years & 3 & 10 & $13(8.55 \%)$ \\
\hline $21-30$ years & 6 & 42 & $48(31.57 \%)$ \\
\hline $31-40$ years & 5 & 34 & $39(25.66 \%)$ \\
\hline $41-50$ years & 6 & 16 & $12(14.47 \%)$ \\
\hline $51-60$ years & 8 & 7 & $10(6.58 \%)$ \\
\hline 61 years and above & 5 & 5 & $152(100 \%)$ \\
\hline Total & $35(23.03 \%)$ & $117(76.97 \%)$ & \\
\hline
\end{tabular}

ABBREVIATIONS : G- Gentamycin, A - Ampicillin, AS -Ampicillin- Sulbactum, AK- Amikacin, CE - Cefotaxime, CA- Ceftazidime, CI - Ceftriaxone, CF - Ciprofloxacin, SXT- TrimethoprimSulphamethoxazole, I - Imipenem, LZ - Linezolid, NA- Nalidixic Acid , NF - Nitrofurantoin, NX Norfloxacin, P - Penicillin , VA - Vancomycin, PT - Piperacillin - Tazobactum, NT - Not tested, ABST - Antibiotic sensitivity testing

ACKNOWLEDGEMENTS: Funding: None. Ethical approval: Not required. I would like to express my sincere thanks to Dr. Raghuram V, Associate Professor Dept. of Community Medicine, KIMS for his contribution during preparation of this manuscript.

\section{REFERENCES:}

1. Fry J. Medicine in Three Societies. Lancaster: Medical andTechnical Publishing Co. Ltd, 1969.

2. Royal College of General Practitioners Office of Population Consensus \& Survey. Department of Health Morbidity Statistics from General Practice, 1991-1992. London: HMSO, 1995.

3. Car J. Urinary tract infections in women: diagnosis and management in primary care. BMJ 2006; 332: 94-7.

4. Foxman B. Epidemiology of urinary tract infections: incidence,morbidity, and economic costs. Am J Med 2002; 113 Suppl 1A: 5-13S.

5. Foxman B, Barlow R, D'Arcy $\mathrm{H}$ et al. Urinary tract infection:self-reported incidence and associated costs. Ann Epidemiol 2000; 10:509-15.

6. Stamm WE, Norrby SR (2001) Urinary tract infections: disease panorama and challenges. J Infect Dis 183 Suppl 1:S1-S4.

7. Gonzalez CM, Schaeffer AJ (1999) Treatment of urinary tract infection: what's old, what`s new, and what works. World J Urol 6:372-382. 
8. Gupta K, Hooten TM, Stamm WE (2001) Increasing antimicrobial resistance and the management of uncomplicated community-acquired urinary tract infections. Ann Intern Med 135:41-50.

9. Nicolle LE (2001) Epidemiology of urinary tract infection. Infect Med 18:153-162.

10. Smith RD , Coast J , 2002 . Antimicrobial resistance: a global response .Bull World Health Organ 80: $126-133$.

11. Schaeffer AJ: The expanding role of fluoroquinolones. Am J Med2002, 113(Suppl 1A):45S-54S.

12. Biswas D, Gupta P, Prasad R, Singh V, Arya M, Kumar A: Choice of antibiotic for empirical therapy of acute cystitis in a setting of high antimicrobial resistance. Indian J Med Sci2006,60(2):53-8.

13. Goldstein FW: Antibiotic susceptibility of bacterial strains isolated from patients with community-acquired urinary tract infections in France. Multicentre Study Group. Eur J ClinMicrobiolInfect Dis 2000, 19:112-117.

14. Gupta V, Yadav A, Joshi RM: Antibiotic resistance pattern in uropathogen. Indian J Med Microbiol2002, 20:96-98.

15. Tankhiwale SS, Jalgaonkar SV, Ahamad S, Hassani U: Evaluation of extended spectrum beta lactamase in urinary isolates. IndianJ Med Res 2004, 120:553-556.

16. Kumar MS, Lakshmi V, Rajagopalan R: Related Articles, Occurrence of extended spectrum beta-lactamases among Enterobacteriaceae spp. isolated at a tertiary care institute.Indian J Med Microbiol2006, 24(3):208-11.

17. Manges AR, Natarajan P, Solberg OD, Dietrich PS, Riley LW: The changing prevalence of drug-resistant Escherichia coli clonal groups in a community: evidence for community outbreaks of urinary tract infections. Epidemiol Infect2006, 134(2):425-31.

18. Kahan NR, Chinitz DP, Waitman DA, Dushnitzky D, Kahan E, Shapiro M:Empiric treatment of uncomplicated urinary tract infection with fluoroquinolones in older women in Israel: another lost treatment option? Ann Pharmacother2006, 40(12):22237.

19. Zhanel GG et al. (2005) Antibiotic resistance in outpatient urinary isolates: final results from the North American Urinary Tract Infection Collaborative Alliance (NAUTICA). Int J Antimicrob Agents 26:380-388.

20. Andrade SS, Sader HS, Jones RN, Pereira AS, Pignatari AC, Gales AC (2006) Increased resistance to first-line agents among bacterial pathogens isolated from urinary tract infections in Latin America: time for local guidelines? MemInstOswaldo Cruz 101:741748.

21. Meharwal SK, Taneja N, Sharma SK, Sharma M: Complicated nosocomialUTI caused by nonfermenters. Indian J Urol2002, 18:123-128.

22. Kumar MS, Lakshmi V, Rajagopalan R: Related Articles, Occurrence of extended spectrum beta-lactamases among Enterobacteriaceae spp. isolated at a tertiary care institute.Indian J Med Microbiol2006, 24(3):208-11.

23. Khan AU, Musharraf A: Plasmid mediated multiple antibiotic resistance in P. mirabilis isolated from the UTI patients. MedicalSci Mon 2004, 10:598-602.

24. Schaeffer AJ ,Rajan N , Cao Q , Anderson BE , Pruden DL, SensibarJ , Duncan JL , 2001. Host pathogenesis in urinary tract infections Int J AntimicrobAgents 17: 245 - 251.

25. Mudur G (2000) Drug resistant cholera in India attributed to antibiotic misuse. BMJ 321:1368-1369.

26. Magee JT, Pritchard EL, Fitzgerald KA, Dunstan FDJ, Howard AJ (1999) Antibiotic prescribing and antibiotic resistance in community practice: retrospective study, 19968. BMJ 319:1239-1240. 
27. McNulty CAM et al. (2006) Clinical relevance of laboratory-reported antibiotic resistance in acute uncomplicated urinary tract infection in primary care. J AntimicrobChemother 58:1000-1008.

28. Gupta K, Stamm WE (2002) Outcomes associated with trimethoprim/sulphamethoxazole (TMP/SMX) therapy in TMP/SMX resistant community-acquired UTI. Int J Antimicrob Agents 19:554-556. 